\section{Fatores associados à carga de doenças da síndrome metabólica entre adultos brasileiros}

\author{
Factors associated with the burden of metabolic \\ syndrome diseases among Brazilian adults
}

\begin{abstract}
This study aimed to identify socio-demographic and behavioral factors associated with the burden of metabolic syndrome diseases, based on data collected in 2007 by the Telephone Survey System for the Surveillance of Risk and Protective Factors for Chronic Non-Communicable Diseases (VIGITEL). The sample included 49,276 adults living in the Brazilian State capitals and Federal District. Metabolic syndrome was defined as the presence of at least two of the following: diabetes, dyslipidemia, high blood pressure, and obesity. Burden of disease showed that 22.7\% of the adult population presented at least one of these conditions and $14.2 \%$ had two or more. Adjusted prevalence ratios showed that the odds of metabolic syndrome increased after 25 years of age, with insufficient physical activity, and with overweight in both men and women. Public health promotion policies should be encouraged that include modifiable factors with a major impact on the prevention and treatment of metabolic syndrome, as well as clinical diagnosis in populations at risk of metabolic syndrome.
\end{abstract}

Metabolic Syndrome X; Adult Health; Surveillance
Naíza Nayla Bandeira de Sá 1,2 Erly Catarina Moura 2,3

\section{Introdução}

Síndrome metabólica trata de um conjunto de alterações fisiopatológicas simultâneas que aumentam o risco de doenças cardiovasculares 1 . As principais alterações fisiopatológicas se referem aos distúrbios metabólicos, tendo origem nos carboidratos, em particular no metabolismo da glicose. Na primeira definição oficial da síndrome metabólica, pela Organização Mundial da Saúde (OMS), a obrigatoriedade da presença de diabetes ou resistência à insulina ou intolerância à glicose ou glicemia de jejum elevada se justificava pela semelhança nos mecanismos fisiopatológicos destas situações, que resultam no aumento da glicemia e na alteração do metabolismo de lipídios, acompanhadas de alterações hormonais, provavelmente em conseqüência do excesso de peso, favorecendo o aparecimento das doenças cardiovasculares 2,3,4,5.

Por conta de outras definições, que consideram também a simultaneidade de algumas alterações fisiopatológicas, entre elas a obesidade (especialmente a central), a dislipidemia (principalmente triglicérides elevado e HDL-colesterol baixo), a hipertensão arterial (hipertensão confirmada ou pressão arterial sistólica e/ou diastólica elevada) e a diabetes (diabetes confirmada ou resistência à insulina ou intolerância à glicose ou glicemia de jejum elevada), todas associadas ao aumento da glicemia 6,7,8,9,10, diferentes instituições americanas estudiosas do 
assunto propuseram recentemente, consenso único e global, que consideram para o diagnóstico da síndrome metabólica pelo menos três das seguintes situações: circunferência da cintura, triglicérides, pressão arterial e glicemia elevadas e HDL-colesterol baixo, ou uso de medicação no caso das quatro últimas situações 11 .

O objetivo principal do diagnóstico da síndrome metabólica é a adequação do tratamento e o controle dos fatores de risco que possam potencializar o quadro, na medida em que as doenças que compõem esta síndrome são crônicas e suas seqüelas irreversíveis 12 . Todavia, o diagnóstico da síndrome metabólica está condicionado à realização de exames físicos e bioquímicos, muitos deles ainda de difícil acesso pela população brasileira. Dessa forma, o uso de informações já existentes, de base populacional, que se aproximam dos indicadores recomendados, é aceitável como triagem para uma primeira estimativa da ocorrência e distribuição da síndrome metabólica no país, no sentido de contribuir para melhor compreensão da sua magnitude, ainda que subestimada pela falta de acesso a serviços diagnósticos, e subsidiar ações de intervenção no sentido de reduzir os fatores de risco a ela associados. O sistema contínuo de Vigilância de Fatores de Risco e Proteção para Doenças Crônicas por Inquérito Telefônico (VIGITEL) implantado em 2006 pela Secretaria de Vigilância em Saúde do Ministério da Saúde, se configura numa importante ferramenta para estudos dessa natureza, uma vez que, dentre as questões realizadas, é possível identificar as quatro situações consensuais recomendadas para a classificação da síndrome metabólica, isto é, indicadores de obesidade, hipertensão arterial, diabetes e dislipidemia 13,14,15.

Este artigo tem como objetivo identificar os fatores sociodemográficos e comportamentais associados à carga de doenças que compõem a síndrome metabólica, junto à população adulta, além de apontar o público a ser priorizado para o diagnóstico desta síndrome, conforme o atual consenso sobre síndrome metabólica 11.

\section{Métodos}

O estudo realizado foi do tipo transversal, com base em dados obtidos pelo VIGITEL, durante o ano de 2007. Trata-se de pesquisa feita por meio de inquérito telefônico, junto a 54.251 indivíduos com 18 e mais anos de idade, residentes nas capitais dos 26 estados brasileiros e no Distrito Federal.

A amostra para o estudo foi obtida em duas etapas. A primeira constou do sorteio sistemático de 5 mil linhas telefônicas fixas residenciais para cada cidade avaliada, que foram ressorteadas e agrupadas em 25 réplicas com 200 números cada. A segunda etapa foi composta pela enumeração dos moradores com 18 ou mais anos de idade, residentes no domicílio e que consentiram em participar da pesquisa. Para poder estimar com coeficiente de $95 \%$ de confiança e erro máximo de cerca de dois pontos percentuais a freqüência de qualquer fator de risco na população estudada, calculou-se um mínimo de 2 mil entrevistas para cada cidade 13,16 .

$\mathrm{O}$ inquérito telefônico constou de entrevista utilizando-se um questionário eletrônico com questões sobre: características sociodemográficas, padrão de alimentação e de atividade física, peso e altura recordados, tabagismo e consumo de bebidas alcoólicas, avaliação do estado de saúde e morbidade.

Para este estudo, peso e altura auto-referidos foram utilizados para o cálculo do índice de massa corporal (IMC), que foi usado para definir obesidade, quando $\geq 30 \mathrm{~kg} / \mathrm{m}^{2}$. Diabetes, dislipidemia (qualquer alteração lipídica) e hipertensão arterial (independentemente dos valores pressóricos isolados) foram consideradas presentes quando havia resposta positiva ao diagnóstico médico anterior destas doenças. Todas essas condições foram consideradas em relação à questão: "Algum médico já lhe disse que o(a) Sr(a). tem diabetes? (ou dislipidemia? ou hipertensão arterial?)". Foram excluídas gestantes, num total de 449 , e 4.526 indivíduos que não informaram peso e/ou altura atuais, restando 49.276 casos para análise.

Para o cálculo da carga de doenças próprias da síndrome metabólica, considerou-se valor 1 para a presença de qualquer das seguintes alterações: diabetes, dislipidemia, hipertensão arterial e obesidade, de modo que a soma variou de 0 a 4. Proxy da síndrome metabólica foi considerada variável desfecho (dependente) na presença de pelo menos duas das quatro situações: diabetes, dislipidemia, hipertensão arterial e/ou obesidade, isto é, quando a carga de doenças fosse $\geq 2$. Idade, escolaridade, união conjugal, cor, região de moradia, atividade física, consumo abusivo de bebidas alcoólicas, hábito de fumar e padrão alimentar foram consideradas como variáveis independentes 16,17.

A idade foi classificada em seis faixas etárias: 18-24, 25-34, 35-44, 45-54, 55-64 ou $\geq 65$ anos; a escolaridade em três faixas: $0-8,9-11$ ou $\geq 12$ anos de estudo; a cor branca em sim ou não; a união conjugal estável em sim ou não; a região de moradia em cinco categorias: Norte, Nordeste, Centro-oeste, Sul e Sudeste. O padrão alimentar foi classificado em três categorias: bom, 
regular e ruim. Padrão alimentar foi considerado bom na ocorrência de consumo de frutas, legumes e verduras $\geq 5$ dias na semana, consumo de feijão $\geq 5$ dias na semana, consumo de refrigerante $\leq 2$ vezes na semana e não hábito de consumo de carne/frango com gordura aparente; regular na ocorrência de duas a três destas situações; e ruim na ocorrência menor ou igual a um. O consumo abusivo de bebidas alcoólicas foi considerado presente quando havia ingestão de mais de cinco doses de bebida alcoólica para o homem e mais de quatro para a mulher numa mesma ocasião, pelo menos uma vez nos últimos 30 dias; a atividade física foi categorizada em suficiente, não suficiente ou insuficiente. Considerou-se atividade física suficiente a prática de pelo menos 30 minutos diários de atividade física de intensidade leve ou moderada, isto é, caminhada, caminhada em esteira, musculação, hidroginástica, ginástica em geral, natação, artes marciais, ciclismo e voleibol em cinco ou mais dias da semana, ou a prática de pelo menos 20 minutos diários de atividade física de intensidade vigorosa, isto é, corrida, corrida em esteira, ginástica aeróbica, futebol, basquetebol e tênis, em três ou mais dias da semana 18 . A atividade física insuficiente foi considerada quando não havia prática de qualquer atividade física no tempo livre nos últimos três meses, não realização de esforço físico intenso no trabalho, não deslocamento para o trabalho a pé ou de bicicleta e não responsabilidade pela limpeza pesada da casa. As demais situações foram consideradas como atividade física não suficiente, incluindo os quatro domínios (tempo livre, ocupação, transporte e trabalho doméstico). Excesso de peso foi considerado quando o IMC (peso em quilos dividido pelo quadrado da altura em metros) fosse $\geq 25 \mathrm{~kg} / \mathrm{m}^{2} 19$; e o hábito de fumar foi categorizado em nunca fumante, ex-fumante ou fumante atual.

Para obter estimativas gerais das variáveis estudadas para o conjunto da população adulta foram utilizados quatro fatores de ponderação: (1) número de adultos em cada residência, (2) inverso do número de linhas telefônicas fixas em cada residência, (3) razão entre a freqüência relativa de indivíduos da amostra estudada e da amostra do Censo Demográfico de 2000 em determinadas categorias sociodemográficas, e (4) fração da amostra estudada em cada cidade em relação à população adulta de cada cidade segundo o Censo Demográfico de 2000. Os quatro fatores são multiplicados e os produtos resultantes são utilizados para calcular os percentuais ponderados para ajustar a distribuição sociodemográfica da amostra VIGITEL à distribuição da população adulta de cada cidade no Censo Demográfico de
2000, levando-se em conta o peso populacional de cada cidade no conjunto das cidades estudadas. Maiores detalhes podem ser vistos em outras publicações 13,16.

Todas as estimativas foram feitas separadamente para cada sexo, considerando-se intervalo de 95\% de confiança (IC95\%). Razões de prevalência (RP), bruta, ajustada para a idade e ajustada para todas as variáveis estudadas (idade, escolaridade, cor, união conjugal, região de moradia, padrão alimentar, consumo abusivo de bebidas alcoólicas, atividade física, excesso de peso e hábito de fumar) foram calculadas por regressão de Poisson. A adoção do excesso de peso (préobesidade + obesidade) para ajuste do modelo se justifica pela sua alta prevalência (mais de $40 \%$ ), ainda que a obesidade (quase $13 \%$ ) faça parte da composição da variável dependente deste estudo. Considera-se que a pré-obesidade é um fator de risco importante para a síndrome metabólica e passível de modificação.

Por se tratar de entrevista por telefone, o consentimento livre e esclarecido foi substituído pelo consentimento verbal, obtido por ocasião dos contatos telefônicos com os entrevistados. Este estudo foi aprovado pela Comissão de Ética em Pesquisa em Seres Humanos do Ministério da Saúde.

\section{Resultados}

A Tabela 1 mostra as características da população estudada conforme o sexo. A maior parte da população tem entre 18 e 44 anos, estudou até oito anos, é de cor não branca, tem união conjugal estável, mora na Região Sudeste, tem um padrão alimentar regular, não consome bebidas alcoólicas de forma abusiva, tem prática de atividade física não suficiente e nunca fumou. Destaca-se que $42,7 \%$ apresentam excesso de peso.

A Tabela 2 mostra que a diabetes atingiu 5\% da população adulta e a obesidade $12,7 \%$, sem diferença entre os sexos. Observa-se que a dislipidemia e a hipertensão arterial foram mais freqüentes entre as mulheres $(18,1 \%$ e $23,7 \%$, respectivamente) do que entre os homens $(13,4 \%$ e $20,2 \%$, respectivamente). A carga de doença mostra que $22,7 \%$ da população adulta apresentam pelo menos uma das doenças utilizadas para classificar a síndrome metabólica e 14,2\% apresentam duas ou mais, critério utilizado neste trabalho para condicionar a presença de síndrome metabólica.

Observa-se que a prevalência de síndrome metabólica entre os homens (Tabela 3) aumenta com a idade, com a piora do padrão alimentar e de atividade física. É maior nas faixas de esco- 
Distribuição (\%) * da população adulta ( $\geq 18$ anos de idade) segundo características sociodemográficas e comportamentais, por sexo. Vigilância de Fatores de Risco e Proteção para Doenças Crônicas por Inquérito Telefônico (VIGITEL), Brasil, 2007.

\begin{tabular}{|c|c|c|c|c|c|c|}
\hline \multirow[t]{2}{*}{$\begin{array}{l}\text { Características sociodemográficas e } \\
\text { comportamentais }\end{array}$} & \multicolumn{2}{|c|}{$\begin{array}{c}\text { Total } \\
(\mathrm{N}=\mathbf{4 9 . 2 7 6})\end{array}$} & \multicolumn{2}{|c|}{$\begin{array}{l}\text { Homens } \\
(n=20.869)\end{array}$} & \multicolumn{2}{|c|}{$\begin{array}{l}\text { Mulheres } \\
\text { (n=28.407) }\end{array}$} \\
\hline & $\%$ & IC95\% & $\%$ & IC95\% & $\%$ & $1 \mathrm{IC95 \%}$ \\
\hline \multicolumn{7}{|l|}{ Idade (anos) } \\
\hline $18-24$ & 20,8 & $19,8-21,8$ & 21,4 & $19,8-23,1$ & 20,1 & $18,9-21,3$ \\
\hline $25-34$ & 25,3 & $24,3-26,2$ & 26,1 & $24,7-27,6$ & 24,4 & $23,2-25,6$ \\
\hline $35-44$ & 22,1 & $21,3-22,9$ & 22,0 & $20,9-23,2$ & 22,2 & $21,2-23,2$ \\
\hline $45-54$ & 15,1 & $14,5-15,7$ & 14,8 & $13,9-15,8$ & 15,4 & $14,6-16,2$ \\
\hline $55-64$ & 8,8 & $8,3-9,3$ & 8,5 & $7,8-9,2$ & 9,1 & $8,5-9,7$ \\
\hline$\geq 65$ & 7,9 & $7,5-8,4$ & 7,1 & $6,5-7,7$ & 8,8 & $8,2-9,4$ \\
\hline \multicolumn{7}{|l|}{ Escolaridade (anos de estudo) } \\
\hline $0-8$ & 50,1 & $49,0-51,1$ & 52,5 & $51,0-54,1$ & 47,6 & $46,3-48,9$ \\
\hline $9-11$ & 31,8 & $31,0-32,6$ & 29,6 & $28,4-30,9$ & 33,9 & $32,8-35,0$ \\
\hline$\geq 12$ & 18,1 & $17,5-18,8$ & 17,8 & $16,9-18,7$ & 18,5 & $17,7-19,3$ \\
\hline Cor não branca & 60,0 & $59,0-61,0$ & 62,8 & $61,3-64,4$ & 57,1 & $55,9-58,4$ \\
\hline União conjugal estável & 52,9 & $51,8-53,9$ & 56,9 & $55,3-58,5$ & 48,9 & $47,6-50,2$ \\
\hline \multicolumn{7}{|l|}{ Região de moradia } \\
\hline Norte & 9,6 & $9,3-10,0$ & 10,1 & $9,6-10,7$ & 9,2 & $8,7-9,6$ \\
\hline Nordeste & 26,0 & $25,2-26,7$ & 25,9 & $24,7-27,0$ & 26,1 & $25,1-27,0$ \\
\hline Centro-oeste & 10,5 & $10,1-10,9$ & 10,6 & $10,0-11,3$ & 10,4 & $9,8-11,0$ \\
\hline Sudeste & 45,7 & $44,6-46,8$ & 45,5 & $43,8-47,2$ & 45,9 & $44,5-47,2$ \\
\hline Sul & 8,2 & $7,8-8,5$ & 7,9 & $7,3-8,4$ & 8,5 & $8,0-8,9$ \\
\hline \multicolumn{7}{|l|}{ Padrão alimentar } \\
\hline Bom & 21,8 & $20,9-22,7$ & 27,1 & $25,6-28,5$ & 16,6 & $15,7-17,6$ \\
\hline Regular & 66,9 & $65,9-67,9$ & 65,1 & $63,6-66,7$ & 68,6 & $67,5-69,8$ \\
\hline Ruim & 11,3 & $10,7-11,9$ & 7,8 & $7,1-8,5$ & 14,7 & $13,8-15,6$ \\
\hline Consumo abusivo de bebidas alcoólicas & 18,5 & $17,6-19,3$ & 27,5 & $26,0-29,0$ & 9,6 & $8,8-10,4$ \\
\hline \multicolumn{7}{|l|}{ Atividade física } \\
\hline Suficiente & 16,1 & $15,4-16,8$ & 19,1 & $17,8-20,3$ & 13,2 & $12,4-14,0$ \\
\hline Não suficiente & 61,2 & $60,2-62,3$ & 53,1 & $51,5-54,7$ & 69,2 & $68,1-70,4$ \\
\hline Insuficiente & 22,6 & $21,8-23,5$ & 27,8 & $26,3-29,3$ & 17,6 & $16,6-18,5$ \\
\hline Excesso de peso & 42,7 & $41,7-43,7$ & 48,3 & $46,7-49,9$ & 37,3 & $36,0-38,5$ \\
\hline \multicolumn{7}{|l|}{ Hábito de fumar } \\
\hline Nunca fumante & 60,8 & $59,8-61,8$ & 53,0 & $51,4-54,6$ & 68,4 & $67,2-69,6$ \\
\hline Ex-fumante & 22,4 & $21,6-23,2$ & 25,8 & $24,5-27,1$ & 19,1 & $18,1-20,1$ \\
\hline Fumante & 16,8 & $15,9-17,7$ & 21,2 & $19,7-22,7$ & 12,5 & $11,6-13,4$ \\
\hline
\end{tabular}

IC95\%: intervalo de 95\% de confiança.

* Percentual ponderado para ajustar a distribuição sociodemográfica da amostra VIGITEL à distribuição da população adulta de cada cidade no Censo Demográfico de 2000 (Instituto Brasileiro de Geografia e Estatística. http://www.ibge.gov.br), levando em conta o peso populacional de cada cidade no conjunto das cidades estudadas.

laridade extremas (0-8 e $\geq 12$ anos de estudo), naqueles com união conjugal estável, com excesso de peso e nos nunca fumantes. Não se encontrou diferença quanto à cor. No que se refere à região de moradia, verificou-se $14,5 \%$ no $\mathrm{Su}$ deste, $13,2 \%$ no Nordeste, $12,1 \%$ no Sul, $11,1 \%$ no
Centro-oeste e 10,9\% no Norte. As RP ajustadas para as variáveis em estudo mostram que a probabilidade de síndrome metabólica é maior entre os homens: mais velhos, a partir dos 25 anos de idade aumenta mais de $80 \%$ em relação aos mais jovens, chegando a mais do que oito vezes 
Freqüência (\%) * de adultos ( $\geq 18$ anos de idade) segundo presença e carga de doença e condição de síndrome metabólica, por sexo. Vigilância de Fatores de Risco e Proteção para Doenças Crônicas por Inquérito Telefônico (VIGITEL), Brasil, 2007.

\begin{tabular}{|c|c|c|c|c|c|c|}
\hline \multirow[t]{2}{*}{ Doença, carga e síndrome metabólica } & \multicolumn{2}{|c|}{ Total } & \multicolumn{2}{|c|}{ Homens } & \multicolumn{2}{|c|}{ Mulheres } \\
\hline & $\%$ & $\mathrm{IC} 95 \%$ & $\%$ & IC95\% & $\%$ & IC95\% \\
\hline Diabetes & 5,0 & $4,6-5,3$ & 4,8 & $4,3-5,4$ & 5,1 & $4,6-5,6$ \\
\hline Dislipidemia & 15,8 & $15,1-16,4$ & 13,4 & $12,5-14,4$ & 18,1 & $17,2-18,9$ \\
\hline Hipertensão arterial & 22,0 & $21,2-22,8$ & 20,2 & $19,0-21,4$ & 23,7 & $22,6-24,7$ \\
\hline Obesidade & 12,7 & $12,0-13,3$ & 13,5 & $12,5-14,6$ & 11,8 & $11,0-12,6$ \\
\hline \multicolumn{7}{|l|}{ Carga de doença } \\
\hline 0 & 63,1 & $62,2-64,1$ & 64,9 & $63,4-66,4$ & 61,4 & $60,1-62,6$ \\
\hline 1 & 22,7 & $21,9-23,5$ & 21,9 & $20,6-23,1$ & 23,5 & $22,4-24,5$ \\
\hline 2 & 10,4 & $9,9-11$ & 9,8 & $9,0-10,6$ & 11,1 & $10,3-11,8$ \\
\hline 3 & 3,2 & $2,9-3,5$ & 3,1 & $2,7-3,6$ & 3,3 & $2,9-3,7$ \\
\hline 4 & 0,5 & $0,4-0,6$ & 0,3 & $0,2-0,4$ & 0,8 & $0,6-1,0$ \\
\hline Condição de síndrome metabólica & 14,2 & $13,6-14,8$ & 13,2 & $12,3-14,2$ & 15,2 & $14,3-16,0$ \\
\hline
\end{tabular}

IC95\%: intervalo de 95\% de confiança.

* Percentual ponderado para ajustar a distribuição sociodemográfica da amostra VIGITEL à distribuição da população adulta de cada cidade no Censo Demográfico de 2000 (Instituto Brasileiro de Geografia e Estatística. http://www.ibge.gov.br),

levando em conta o peso populacional de cada cidade no conjunto das cidades estudadas.

após 55 anos; que consomem bebidas alcoólicas de forma abusiva; com atividade física insuficiente; com excesso de peso e ex-fumantes. Após o ajuste, escolaridade, união conjugal, região de moradia e padrão alimentar perdem a significância. Cor não se mostrou associada à condição de síndrome metabólica.

Para as mulheres (Tabela 4), assim como para os homens, a prevalência de síndrome metabólica aumenta com a idade, porém reduz com o aumento da escolaridade. É maior naquelas com união conjugal estável, com padrão alimentar ruim, que não consomem bebidas alcoólicas de forma abusiva, que têm atividade física insuficiente, excesso de peso e nas nunca fumantes. Não se encontrou diferença quanto à cor. No que se refere à região de moradia, verificou-se 16,6\% no Nordeste, $16,2 \%$ no Sul, $15,4 \%$ no Sudeste, $12,9 \%$ no Centro-oeste e $11,8 \%$ no Norte. As RP ajustadas para as demais variáveis mostram que a probabilidade de síndrome metabólica é maior a partir dos 25 anos de idade, quando atinge mais do que o dobro da probabilidade encontrada entre as mais jovens, é maior entre as mulheres com atividade física insuficiente e com excesso de peso. $\mathrm{O}$ aumento da escolaridade se comporta como fator de proteção contra a síndrome metabólica. A Região Nordeste apresenta 19\% mais chances de condição de síndrome metabólica do que as demais regiões. Cor, união conjugal, padrão alimentar, consumo abusivo de bebidas alcoólicas e tabagismo não se associaram com condição de síndrome metabólica.

\section{Discussão}

O consenso universal sobre a definição da síndrome metabólica 11 aponta o uso de indicadores de alteração do metabolismo dos carboidratos e dos lipídios, além dos níveis pressóricos e excesso de gordura corporal, considerando-se a presença de três alterações no conjunto de cinco possibilidades: circunferência da cintura elevada para a população de estudo, triglicérides $\geq 150 \mathrm{mg} / \mathrm{dL}$, HDL-colesterol $<40 \mathrm{mg} / \mathrm{dL}$ para homens e $<50 \mathrm{mg} / \mathrm{dL}$ para mulheres, pressão arterial sistólica $\geq 130 \mathrm{mmHg}$ e/ou diastólica $\geq$ $85 \mathrm{mmHg}$ e glicemia de jejum $\geq 100 \mathrm{mg} / \mathrm{dL}$ (ou uso de medicação para controle das últimas quatro possibilidades).

Neste trabalho, adotando-se a combinação de pelo menos duas alterações das quatro estudadas (diabetes, dislipidemia, hipertensão arterial e obesidade), a síndrome metabólica atingiu $14,2 \%$ da população pesquisada, sendo a maior prevalência encontrada entre as mulheres (15,2\%) quando comparadas aos homens $(13,2 \%)$. Após ajustes, verificou-se que os fatores de risco identificados para a síndrome metabólica foram maior idade, atividade física insuficiente e excesso de peso em ambos os sexos. Entre os 
Tabela 3

Prevalência (\%) * e razões de prevalência (RP) de condição de síndrome metabólica em homens ( $\geq 18$ anos de idade) segundo características sociodemográficas e comportamentais. Vigilância de Fatores de Risco e Proteção para Doenças Crônicas por Inquérito Telefônico (VIGITEL), Brasil, 2007.

\begin{tabular}{|c|c|c|c|c|c|c|c|c|}
\hline \multirow[t]{2}{*}{$\begin{array}{l}\text { Características sociodemográficas } \\
\text { e comportamentais }\end{array}$} & \multicolumn{2}{|c|}{ Prevalência } & \multicolumn{2}{|c|}{ RP bruta } & \multicolumn{2}{|c|}{ RP ajustada para a idade } & \multicolumn{2}{|c|}{$\begin{array}{c}\text { RP ajustada para todas } \\
\text { as características ** }\end{array}$} \\
\hline & $\%$ & IC95\% & $\%$ & IC95\% & $\%$ & IC95\% & $\%$ & IC95\% \\
\hline \multicolumn{9}{|l|}{ Idade (anos) } \\
\hline $18-24$ & 2,3 & $1,1-3,4$ & 1,00 & & 1,00 & & 1,00 & \\
\hline $25-34$ & 5,9 & $4,5-7,2$ & 2,59 & $1,47-4,55$ & 2,59 & $1,47-4,55$ & 1,86 & $1,05-3,30$ \\
\hline $35-44$ & 12,0 & $10,2-13,8$ & 5,28 & $3,08-9,05$ & 5,28 & $3,08-9,05$ & 3,38 & $1,97-5,81$ \\
\hline $45-54$ & 24,4 & $21,3-27,5$ & 10,74 & $6,31-18,29$ & 10,74 & $6,31-18,29$ & 6,20 & $3,60-10,68$ \\
\hline $55-64$ & 32,2 & $28,2-36,2$ & 14,18 & $8,33-24,13$ & 14,18 & $8,33-24,13$ & 8,35 & $4,88-14,3$ \\
\hline$\geq 65$ & 31,3 & $27,3-35,3$ & 13,77 & $8,08-23,46$ & 13,77 & $8,08-23,46$ & 8,79 & $5,19-14,89$ \\
\hline \multicolumn{9}{|l|}{ Escolaridade (anos de estudo) } \\
\hline $0-8$ & 14,7 & $13,2-16,3$ & 1,00 & & 1,00 & & 1,00 & \\
\hline $9-11$ & 9,8 & $8,7-10,9$ & 0,67 & $0,57-0,78$ & 0,95 & $0,82-1,11$ & 0,93 & $0,80-1,08$ \\
\hline$\geq 12$ & 14,6 & $12,7-16,4$ & 0,99 & $0,84-1,17$ & 1,00 & $0,86-1,17$ & 0,93 & $0,79-1,10$ \\
\hline \multicolumn{9}{|l|}{ Cor } \\
\hline Branca & 12,6 & $11,5-13,8$ & 1,00 & & 1,00 & & 1,00 & \\
\hline Não branca & 14,2 & $12,6-15,9$ & 1,13 & $0,97-1,3$ & 0,99 & $0,87-1,13$ & 0,96 & $0,83-1,11$ \\
\hline \multicolumn{9}{|l|}{ União conjugal } \\
\hline Não estável & 7,0 & $5,8-8,1$ & 1,00 & & 1,00 & & 1,00 & \\
\hline Estável & 18,0 & $16,6-19,4$ & 2,58 & $2,16-3,09$ & 1,23 & $1,05-1,45$ & 1,13 & $0,96-1,33$ \\
\hline \multicolumn{9}{|l|}{ Região de moradia } \\
\hline Norte & 10,9 & $9,6-12,2$ & 1,00 & & 1,00 & & 1,00 & \\
\hline Nordeste & 13,2 & $11,9-14,5$ & 1,21 & $1,04-1,42$ & 1,12 & $0,97-1,29$ & 1,17 & $1,01-1,35$ \\
\hline Centro-oeste & 11,1 & $9,1-13,0$ & 1,02 & $0,82-1,26$ & 0,95 & $0,77-1,18$ & 1,02 & $0,82-1,26$ \\
\hline Sudeste & 14,5 & $12,6-16,3$ & 1,33 & $1,12-1,59$ & 1,09 & $0,93-1,29$ & 1,15 & $0,97-1,36$ \\
\hline Sul & 12,1 & $10,5-13,8$ & 1,12 & $0,93-1,34$ & 0,93 & $0,79-1,10$ & 0,96 & $0,81-1,15$ \\
\hline \multicolumn{9}{|l|}{ Padrão alimentar } \\
\hline Bom & 9,8 & $8,1-11,6$ & 1,00 & & 1,00 & & 1,00 & \\
\hline Regular & 14,2 & $13,0-15,3$ & 1,44 & $1,19-1,76$ & 1,07 & $0,89-1,28$ & 1,07 & $0,89-1,29$ \\
\hline Ruim & 17,4 & $13,8-21,0$ & 1,77 & $1,35-2,33$ & 0,96 & $0,74-1,24$ & 1,00 & $0,77-1,3$ \\
\hline \multicolumn{9}{|c|}{ Consumo abusivo de bebidas alcoólicas } \\
\hline Não & 13,4 & $12,3-14,5$ & 1,00 & & 1,00 & & 1,00 & \\
\hline $\operatorname{Sim}$ & 12,9 & $11,1-14,7$ & 0,97 & $0,82-1,14$ & 1,35 & $1,16-1,57$ & 1,30 & $1,12-1,51$ \\
\hline \multicolumn{9}{|l|}{ Atividade física } \\
\hline Suficiente & 9,8 & $8,3-11,4$ & 1,00 & & 1,00 & & 1,00 & \\
\hline Não suficiente & 12,5 & $11,1-13,8$ & 1,27 & $1,05-1,54$ & 1,14 & $0,96-1,36$ & 1,16 & $0,97-1,37$ \\
\hline Insuficiente & 17,0 & $15,0-19,0$ & 1,73 & $1,42-2,11$ & 1,4 & $1,17-1,67$ & 1,35 & $1,14-1,60$ \\
\hline \multicolumn{9}{|l|}{ Excesso de peso } \\
\hline Não & 5,5 & $4,5-6,4$ & 1,00 & & 1,00 & & 1,00 & \\
\hline $\operatorname{Sim}$ & 21,5 & $19,9-23,1$ & 3,92 & $3,25-4,74$ & 3,10 & $2,58-3,72$ & 2,98 & $2,48-3,58$ \\
\hline \multicolumn{9}{|l|}{ Hábito de fumar } \\
\hline Nunca fumante & 23,0 & $20,8-25,3$ & 1,00 & & 1,00 & & 1,00 & \\
\hline Ex-fumante & 10,2 & $8,1-12,2$ & 2,38 & $2,05-2,75$ & 1,41 & $1,22-1,62$ & 1,33 & $1,16-1,54$ \\
\hline Fumante & 9,7 & $8,6-10,8$ & 1,05 & $0,83-1,32$ & 0,92 & $0,73-1,15$ & 0,97 & $0,77-1,22$ \\
\hline
\end{tabular}

IC95\%: intervalo de 95\% de confiança.

* Percentual ponderado para ajustar a distribuição sociodemográfica da amostra VIGITEL à distribuição da população adulta de cada cidade no Censo Demográfico de 2000 (Instituto Brasileiro de Geografia e Estatística. http://www.ibge.gov.br), levando em conta o peso populacional de cada cidade no conjunto das cidades estudadas;

** Idade, escolaridade, cor, união conjugal, região de moradia, padrão alimentar, consumo abusivo de bebidas alcoólicas, atividade física, excesso de peso e hábito de fumar. 
Tabela 4

Prevalência (\%) * e razões de prevalência (RP) de condição de síndrome metabólica em mulheres ( $\geq 18$ anos de idade) segundo características sociodemográficas e comportamentais. Vigilância de Fatores de Risco e Proteção para Doenças Crônicas por Inquérito Telefônico (VIGITEL), Brasil, 2007.

\begin{tabular}{|c|c|c|c|c|c|c|c|c|}
\hline \multirow[t]{2}{*}{$\begin{array}{l}\text { Características sociodemográficas } \\
\text { e comportamentais }\end{array}$} & \multicolumn{2}{|c|}{ Prevalência } & \multicolumn{2}{|c|}{ RP bruta } & \multicolumn{2}{|c|}{ RP ajustada para a idade } & \multicolumn{2}{|c|}{$\begin{array}{l}\text { RP ajustada para todas } \\
\text { as características ** }\end{array}$} \\
\hline & $\%$ & IC95\% & $\%$ & IC95\% & $\%$ & IC95\% & $\%$ & IC95\% \\
\hline \multicolumn{9}{|l|}{ Idade (anos) } \\
\hline $18-24$ & 2,0 & $1,3-2,8$ & 1,00 & & 1,00 & & 1,00 & \\
\hline $25-34$ & 6,0 & $4,6-7,5$ & 2,99 & $1,91-4,66$ & 2,99 & $1,91-4,66$ & 2,33 & $1,51-3,59$ \\
\hline $35-44$ & 10,9 & $9,3-12,6$ & 5,43 & $3,62-8,14$ & 5,43 & $3,62-8,14$ & 3,69 & $2,46-5,53$ \\
\hline $45-54$ & 23,5 & $21,2-25,8$ & 11,65 & $7,91-17,17$ & 11,65 & $7,91-17,17$ & 7,06 & $4,74-10,53$ \\
\hline $55-64$ & 38,0 & $34,8-41,2$ & 18,84 & $12,83-27,68$ & 18,84 & $12,83-27,68$ & 9,91 & $6,64-14,78$ \\
\hline$\geq 65$ & 43,1 & $39,4-46,7$ & 21,34 & $14,53-31,35$ & 21,34 & $14,53-31,35$ & 11,22 & $7,52-16,73$ \\
\hline \multicolumn{9}{|l|}{ Escolaridade (anos de estudo) } \\
\hline $0-8$ & 21,7 & $20,1-23,3$ & 1,00 & & 1,00 & & 1,00 & \\
\hline $9-11$ & 9,9 & $8,9-10,9$ & 0,46 & $0,41-0,52$ & 0,78 & $0,69-0,89$ & 0,83 & $0,74-0,94$ \\
\hline$\geq 12$ & 8,0 & $7,0-9,0$ & 0,37 & $0,32-0,42$ & 0,51 & $0,44-0,59$ & 0,56 & $0,49-0,65$ \\
\hline \multicolumn{9}{|l|}{ Cor } \\
\hline Branca & 15,4 & $14,2-16,5$ & 1,00 & & 1,00 & & 1,00 & \\
\hline Não branca & 14,9 & $13,6-16,1$ & 0,97 & $0,87-1,08$ & 0,87 & $0,79-0,96$ & 0,98 & $0,89-1,09$ \\
\hline \multicolumn{9}{|l|}{ União conjugal } \\
\hline Não estável & 13,2 & $12,2-14,2$ & 1,00 & & 1,00 & & 1,00 & \\
\hline Estável & 17,2 & $15,9-18,6$ & 1,30 & $1,17-1,46$ & 1,15 & $1,04-1,28$ & 1,05 & $0,95-1,16$ \\
\hline \multicolumn{9}{|l|}{ Região de moradia } \\
\hline Norte & 11,8 & $10,5-13,2$ & 1,00 & & 1,00 & & 1,00 & \\
\hline Nordeste & 16,6 & $15,4-17,8$ & 1,40 & $1,22-1,60$ & 1,19 & $1,05-1,35$ & 1,19 & $1,05-1,34$ \\
\hline Centro-oeste & 12,9 & $11,4-14,3$ & 1,09 & $0,93-1,27$ & 1,00 & $0,87-1,15$ & 0,99 & $0,86-1,14$ \\
\hline Sudeste & 15,4 & $13,7-17,0$ & 1,30 & $1,11-1,51$ & 0,94 & $0,81-1,08$ & 0,95 & $0,83-1,10$ \\
\hline Sul & 16,2 & $14,4-18,0$ & 1,37 & $1,17-1,60$ & 1,00 & $0,86-1,16$ & 1,03 & $0,89-1,20$ \\
\hline \multicolumn{9}{|l|}{ Padrão alimentar } \\
\hline Bom & 9,6 & $8,0-11,2$ & 1,00 & & 1,00 & & 1,00 & \\
\hline Regular & 14,7 & $13,7-15,7$ & 1,53 & $1,28-1,83$ & 0,99 & $0,84-1,18$ & 1,03 & $0,87-1,22$ \\
\hline Ruim & 23,5 & $20,8-26,3$ & 2,45 & $2,00-3,00$ & 1,12 & $0,92-1,37$ & 1,19 & $0,98-1,44$ \\
\hline \multicolumn{9}{|l|}{ Consumo abusivo de bebidas alcoólicas } \\
\hline Não & 15,6 & $14,7-16,5$ & 1,00 & & 1,00 & & 1,00 & \\
\hline Sim & 11,2 & $8,3-14,0$ & 0,72 & $0,55-0,93$ & 1,15 & $0,89-1,49$ & 1,14 & $0,89-1,47$ \\
\hline \multicolumn{9}{|l|}{ Atividade física } \\
\hline Suficiente & 14,6 & $12,7-16,5$ & 1,00 & & 1,00 & & 1,00 & \\
\hline Não suficiente & 13,9 & $12,8-14,9$ & 0,95 & $0,82-1,10$ & 1,05 & $0,91-1,21$ & 1,06 & $0,92-1,22$ \\
\hline Insuficiente & 20,8 & $18,6-23,0$ & 1,42 & $1,20-1,69$ & 1,16 & $0,99-1,36$ & 1,24 & $1,07-1,45$ \\
\hline \multicolumn{9}{|l|}{ Excesso de peso } \\
\hline Não & 6,2 & $5,5-6,8$ & 1,00 & & 1,00 & & 1,00 & \\
\hline Sim & 30,3 & $28,5-32,1$ & 4,92 & $4,35-5,56$ & 3,29 & $2,90-3,73$ & 3,14 & $2,77-3,56$ \\
\hline \multicolumn{9}{|l|}{ Hábito de fumar } \\
\hline Nunca fumante & 14,1 & $13,2-15,1$ & 1,00 & & 1,00 & & 1,00 & \\
\hline Ex-fumante & 21,4 & $19,2-23,7$ & 1,51 & $1,33-1,72$ & 1,11 & $0,98-1,25$ & 1,05 & $0,93-1,18$ \\
\hline Fumante & 11,2 & $9,2-13,2$ & 0,79 & $0,66-0,96$ & 0,70 & $0,58-0,85$ & 0,81 & $0,67-0,97$ \\
\hline
\end{tabular}

IC95\%: intervalo de 95\% de confiança.

* Percentual ponderado para ajustar a distribuição sociodemográfica da amostra VIGITEL à distribuição da população adulta de cada cidade no Censo Demográfico de 2000 (Instituto Brasileiro de Geografia e Estatística. http://www.ibge.gov.br), levando em conta o peso populacional de cada cidade no conjunto das cidades estudadas;

** Idade, escolaridade, cor, união conjugal, região de moradia, padrão alimentar, consumo abusivo de bebidas alcoólicas, atividade física, excesso de peso e hábito de fumar. 
homens, consumo abusivo de bebidas alcoólicas e ser ex-fumante se associaram positivamente com síndrome metabólica. Entre as mulheres, a alta escolaridade se configurou como fator de proteção.

Estudo conduzido por Ford et al. 20, entre os anos de 1988 e 1994, com os participantes adultos ( $\geq 20$ anos de idade) do terceiro inquérito nacional de saúde e nutrição dos Estados Unidos, apontou 21,8\% de síndrome metabólica. A diferença do resultado encontrado pelos autores com o apontado neste trabalho pode ser explicada pelo critério utilizado para a caracterização da síndrome metabólica, uma vez que esses autores definiram esta síndrome segundo os critérios do Adult Treatment Panel III 7, que determina a presença de pelo menos três dos seguintes indicadores: diabetes, hipertensão arterial, dislipidemia (podendo incluir triglicérides e HDL-colesterol separadamente) e obesidade. Outra hipótese pode ser pela conhecida alta prevalência de obesidade na população americana 21 .

Carnethon et al. 22 em estudo do tipo coorte, realizado de 1985 a 2001, com adultos de 18 a 30 anos de idade, nos Estados Unidos, apontaram que o risco relativo (RR) de síndrome metabólica aumenta com a idade, sendo ainda maior entre os indivíduos negros e com menor escolaridade, do mesmo modo que identificamos no nosso trabalho quanto à idade e à escolaridade, este último entre as mulheres. Após ajuste para idade, raça e sexo, o RR aumenta ainda mais para os indivíduos com IMC elevado. Os autores apontaram como conclusão do estudo que o IMC e o ganho de peso são importantes fatores de risco para a síndrome metabólica.

Ford et al. 23 conduziram estudo junto a 1.626 indivíduos com 20 ou mais anos de idade, participantes do inquérito nacional de saúde e nutrição dos Estados Unidos (1999-2000), identificando que a síndrome metabólica (segundo definição do National Cholesterol Education Program) foi mais prevalente entre os indivíduos com maior idade, dado semelhante ao encontrado neste trabalho.

Os fatores de risco para a síndrome metabólica são o conjunto de fatores das doenças crônicas não transmissíveis, salientando-se os determinantes sociodemográficos e comportamentais, como a dieta, o consumo abusivo de bebidas alcoólicas, a atividade física e o tabagismo 12. Contraditoriamente, no nosso estudo, o padrão alimentar nos dois sexos, o não consumo abusivo de bebidas alcoólicas e atividade física não suficiente/insuficiente e o hábito de fumar entre as mulheres não foram identificados como fatores de risco para a síndrome metabólica.
As limitações principais deste estudo foram: a técnica de coleta de dados utilizada (inquérito telefônico), pois exclui indivíduos que residem em domicílios sem telefone fixo, embora tenham sido utilizados pesos para corrigir a super ou subrepresentação da amostra, tendo como referência a composição sociodemográfica da população adulta do país no censo mais recente; o uso do diagnóstico médico auto-referido de diabetes, dislipidemia e hipertensão arterial e não do diagnóstico baseado em avaliação clínica, física e bioquímica; a estimativa da obesidade baseada em peso e altura auto-referidos e não aferidos, além do que muitos recomendam o uso da circunferência da cintura e não o IMC, todavia, a literatura tem mostrado alta correlação entre IMC e circunferência da cintura para o diagnóstico da obesidade 24; a limitação a apenas quatro das cinco possibilidades de alterações utilizadas para o diagnóstico da síndrome metabólica, o que procuramos minimizar utilizando o corte em duas alterações e não em três; o fato de ser um estudo do tipo transversal, uma vez que a coleta de dados é feita no mesmo momento, espaço e tempo, não podendo distinguir causa - efeito do problema.

Peixoto et al. ${ }^{25}$, em estudo transversal de base populacional, realizado em Goiânia, capital do Estado de Goiás, no ano de 2001, junto a 1.023 indivíduos de 20 a 64 anos, constataram que os resultados encontrados para a prevalência de obesidade com peso e altura referidos foram semelhantes aos obtidos pelo peso e altura aferidos, o que torna confiável, também, os dados apresentados neste trabalho.

O aumento da obesidade na população mundial impacta na maior prevalência de síndrome metabólica 5,22 , pois o aumento do peso ponderal representa um importante fator de risco para as doenças crônicas não transmissíveis, uma vez que as implicações desta condição predizem o surgimento de doenças cardiovasculares, hipertensão e diabetes 26 , ou seja, doenças próprias da síndrome metabólica.

As doenças próprias da síndrome metabólica podem ser uma importante conseqüência da obesidade que atinge atualmente quase $13 \%$ da população brasileira 15. Ressalta-se, no entanto, a importância de avaliar a ocorrência da síndrome metabólica e os fatores a ela associados, de modo a poder contribuir na formulação de políticas públicas de promoção da saúde, considerando especialmente a alimentação e a atividade física, fatores modificáveis que têm grande impacto na prevenção e tratamento dessas doenças.

Este estudo indica a possibilidade de ocorrência de síndrome metabólica em pelo menos 
14\% da população adulta brasileira, com maiores prevalências para os indivíduos mais idosos, com atividade física insuficiente e com excesso de peso; para as mulheres de baixa escolaridade e para os homens com consumo abusivo de bebi- das alcoólicas e ex-fumantes. Esses dados devem ser entendidos como uma possível triagem para priorização do diagnóstico de síndrome metabólica pelas unidades de saúde, conforme recomenda o consenso atual 11.

\section{Resumo}

O objetivo deste trabalho é identificar fatores sociodemográficos e comportamentais associados à carga de doenças da síndrome metabólica, com base em dados coletados em 2007 pelo sistema de Vigilância de Fatores de Risco e Proteção para Doenças Crônicas por Inquérito Telefônico (VIGITEL). Foram avaliados 49.276 adultos, residentes nas capitais dos estados brasileiros e no Distrito Federal. Síndrome metabólica foi considerada na presença de pelo menos duas situações entre diabetes, dislipidemia, hipertensão arterial e obesidade. A carga de doença mostra que 22,7\% da população adulta apresentam pelo menos uma das situações anteriormente descritas e 14,2\% duas ou mais. As razões de prevalência ajustadas mostram que a probabilidade de síndrome metabólica é maior a partir dos 25 anos de idade, na atividade física insuficiente e na presença de excesso de peso nos dois sexos. Políticas públicas de promoção da saúde, que incorporem fatores modificáveis, que têm grande impacto na prevenção e tratamento da síndrome metabólica, devem ser estimuladas, bem como o diagnóstico clínico de populações de risco.

Síndrome X Metabólica; Saúde do Adulto; Vigilância

\section{Colaboradores}

N. N. B. Sá e E. C. Moura contribuíram igualmente na elaboração deste artigo.

\section{Agradecimentos}

À Secretaria de Vigilância em Saúde, Ministério da Saúde, pela cessão do banco de dados. 


\section{Referências}

1. Grundy SM, Cleeman JI, Daniels SR, Donato KA, Eckel RH, Franklin BA, et al. Diagnosis and management of the metabolic syndrome. Circulation 2005; 112:285-9.

2. Matos AFG, Moreira RO, Guedes EP. Aspectos neuroendócrinos da síndrome metabólica. Arq Bras Endocrinol Metab 2003; 47:410-21.

3. Bahia L, Aguiar LGK, Villela NR, Bottino D, Bouskela E. O endotélio na síndrome metabólica. Arq Bras Endocrinol Metab 2006; 50:291-303.

4. De Caterina R, Zampolli A, Del Turco S, Madonna R, Massaro M. Nutritional mechanisms that influence cardiovascular disease. Am J Clin Nutr 2006; 83 Suppl:421S-6S.

5. Ferrari CKB. Atualização: fisiopatologia e clínica da síndrome metabólica. ACM Arq Catarin Med 2007; 36:90-5.

6. World Health Organization. Definition, diagnosis and classification of diabetes mellitus and its complications. Geneva: World Health Organization; 1999.

7. Expert Panel on Detection, Evaluation, and Treatment of High Blood Cholesterol in Adults. Executive Summary of The Third Report of The National Cholesterol Education Program (NCEP) Expert Panel on Detection, Evaluation, And Treatment of High Blood Cholesterol In Adults (Adult Treatment Panel III). JAMA 2001; 285:2486-97.

8. International Diabetes Federation. The IDF consensus worldwide definition of the metabolic syndrome. http://www.bibalex.org/supercourse/ metabolic/IDF1.pdf (acessado em 15/Ago/2009).

9. Sociedade Brasileira de Hipertensão/Sociedade Brasileira de Cardiologia/Sociedade Brasileira de Endocrinologia e Metabologia/Sociedade Brasileira de Diabetes/Associação Brasileira para Estudos da Obesidade. I Diretriz brasileira de diagnóstico e tratamento da síndrome metabólica. Arq Bras Cardiol 2005; 84 Suppl 1:3-28.

10. Sposito AC, Caramelli B, Fonseca FAH, Bertolami MC, Afiune Neto A, Souza AD, et al. IV Diretriz brasileira sobre dislipidemias e prevenção da aterosclerose: Departamento de Aterosclerose da Sociedade Brasileira de Cardiologia. Arq Bras Cardiol 2007; 88 Suppl 1:2-19.

11. Alberti KGMM, Eckel RH, Grundy SM, Zimmet PZ, Cleeman JI, Donato KA, et al. Harmonizing the metabolic syndrome. Circulation 2009; 120:1640-5.

12. World Health Organization. The World Health Report: reducing risks, promoting healthy life. Geneva: World Health Organization; 2002.

13. Ministério da Saúde. VIGITEL 2006: vigilância de fatores de risco e proteção para doenças crônicas por inquérito telefônico. Brasília: Ministério da Saúde; 2007.
14. Ministério da Saúde. VIGITEL 2007: vigilância de fatores de risco e proteção para doenças crônicas por inquérito telefônico. Brasília: Ministério da Saúde; 2008.

15. Ministério da Saúde. VIGITEL 2008: vigilância de fatores de risco e proteção para doenças crônicas por inquérito telefônico. Brasília: Ministério da Saúde; 2009.

16. Moura EC, Malta DC, Morais Neto OL, Monteiro CA. Prevalence and social distribution of risk factors for chronic noncommunicable diseases in Brazil. Rev Panam Salud Pública 2009; 26:17-22.

17. Barreto SM, Passos VMA, Giatti L. Comportamento saudável entre adultos jovens no Brasil. Rev Saúde Pública 2009; 43 Suppl 2:9-17.

18. Florindo AA, Hallal PC, Moura EC, Malta DC. Prática de atividades físicas e fatores associados em adultos, Brasil, 2006. Rev Saúde Pública 2009; 43 Suppl 2:65-73.

19. World Health Organization. Physical status: the use and interpretation of anthropometry. Geneva: World Health Organization; 1995.

20. Ford ES, Giles WH, Dietz WH. Prevalence of the metabolic syndrome among us adults: findings from the Third National Health and Nutrition Examination Survey. JAMA 2002; 287:356-9.

21. National Center for Health Statistics, Centers for Disease Control and Prevention. Prevalence of overweight and obesity among adults: United States, 1999-2002. http://www.cdc.gov/nchs/ products/pubs/pubd/hestats/obese/obse99.htm (acessado em 10/Set/2009).

22. Carnethon MR, Loria CM, Hill JO, Sidney S, Savage PJ, Liu K. Risk factors for the metabolic syndrome: the coronary artery risk development in young adults (CARDIA) study, 1985-2001. Diabetes Care 2004; 27:2707-15.

23. Ford ES, Kohl 3rd HW, Mokdad AH, Ajani UA. Sedentary behavior, physical activity, and the metabolic syndrome among U.S. adults. Obes Res 2005; 13:608-14.

24. Castro SH, Matos HJ, Gomes MB. Parâmetros antropométricos e síndrome metabólica em diabetes tipo 2. Arq Bras Endocrinol Metab 2006; 50:450-5.

25. Peixoto MRG, Benício MHDA, Jardim PCBV. Validade do peso e altura referidos: o estudo de Goiânia. Rev Saúde Pública 2006; 40:1065-72.

26. World Health Organization. Diet, nutrition and the prevention of chronic diseases: report of a joint WHO/FAO. Geneva: World Health Organization; 2003.

Recebido em 12/Set/2009

Versão final reapresentada em 10/Abr/2010

Aprovado em 14/Jun/2010 